\title{
ORIGINAL
}

\section{Lung ultrasound for the early diagnosis of COVID-19 pneumonia: an international multicenter study}

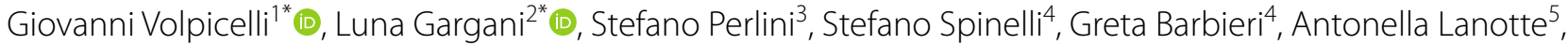
Gonzalo García Casasola ${ }^{6}$, Ramon Nogué-Bou7, Alessandro Lamorte ${ }^{8}$, Eustachio Agricola ${ }^{9,10}$, Tomas Villén ${ }^{11}$, Paramjeet Singh Deol ${ }^{12}$, Peiman Nazerian ${ }^{13}$, Francesco Corradi ${ }^{14,15}$, Valerio Stefanone ${ }^{16}$, Denise Nicole Fraga ${ }^{17}$, Paolo Navalesi ${ }^{18}$, Robinson Ferre ${ }^{19}$, Enrico Boero ${ }^{20}$, Giampaolo Martinelli ${ }^{21}$, Lorenzo Cristoni ${ }^{22}$, Cristiano Perani ${ }^{23}$, Luigi Vetrugno ${ }^{24}$, Cian McDermott ${ }^{25}$, Francisco Miralles-Aguiar ${ }^{26}$, Gianmarco Secco ${ }^{3}$, Caterina Zattera ${ }^{3}$, Francesco Salinaro ${ }^{3}$, Alice Grignaschi ${ }^{5}$, Andrea Boccatonda ${ }^{5}$, Fabrizio Giostra ${ }^{5}$, Marta Nogué Infante ${ }^{7}$, Michele Covella ${ }^{8}$, Giacomo Ingallina ${ }^{10}$, Julia Burkert ${ }^{12}$, Paolo Frumento ${ }^{27}$, Francesco Forfori ${ }^{14}$ and Lorenzo Ghiadoni ${ }^{4}$ on behalf of the International Multicenter Study Group on LUS in COVID-19

\begin{abstract}
Purpose: To analyze the application of a lung ultrasound (LUS)-based diagnostic approach to patients suspected of COVID-19, combining the LUS likelihood of COVID-19 pneumonia with patient's symptoms and clinical history.

Methods: This is an international multicenter observational study in 20 US and European hospitals. Patients suspected of COVID-19 were tested with reverse transcription-polymerase chain reaction (RT-PCR) swab test and had an LUS examination. We identified three clinical phenotypes based on pre-existing chronic diseases (mixed phenotype), and on the presence (severe phenotype) or absence (mild phenotype) of signs and/or symptoms of respiratory failure at presentation. We defined the LUS likelihood of COVID-19 pneumonia according to four different patterns: high (HighLUS), intermediate (IntLUS), alternative (AltLUS), and low (LowLUS) probability. The combination of patterns and phenotypes with RT-PCR results was described and analyzed.

Results: We studied 1462 patients, classified in mild $(n=400)$, severe $(n=727)$, and mixed $(n=335)$ phenotypes. HighLUS and IntLUS showed an overall sensitivity of 90.2\% (95\% Cl 88.23-91.97\%) in identifying patients with positive RT-PCR, with higher values in the mixed (94.7\%) and severe phenotype (97.1\%), and even higher in those patients
\end{abstract}

\footnotetext{
*Correspondence: giovi.volpicelli@gmail.com; gargani@ifc.cnr.it 1 Department of Emergency Medicine, San Luigi Gonzaga University Hospital, Regione Gonzole 10, Orbassano, 10024 Turin, Italy 2 Institute of Clinical Physiology, National Research Council, Via Moruzzi 1, 56124 Pisa, Italy

Full author information is available at the end of the article
}

Giovanni Volpicelli and Luna Gargani are joint first authors and have equally contributed to this work.

The collaborators members of the International Multicenter Study Group on LUS in COVID-19 are listed in "Acknowledgements".

\section{实 Springer}


with objective respiratory failure (99.3\%). The HighLUS showed a specificity of 88.8\% (Cl 85.55-91.65\%) that was higher in the mild phenotype (94.4\%; Cl 90.0-97.0\%). At multivariate analysis, the HighLUS was a strong independent predictor of RT-PCR positivity (odds ratio 4.2, confidence interval 2.6-6.7, $p<0.0001$ ).

Conclusion: Combining LUS patterns of probability with clinical phenotypes at presentation can rapidly identify those patients with or without COVID-19 pneumonia at bedside. This approach could support and expedite patients' management during a pandemic surge.

Keywords: Lung ultrasound, SARS-CoV-2, COVID-19, Interstitial pneumonia

\section{Introduction}

Since the beginning of the SARS-CoV-2 pandemic, frontline physicians with experience in lung ultrasound (LUS) have brought this technique into the spotlight as an efficient tool to diagnose interstitial pneumonia associated with coronavirus disease 2019 (COVID-19) [1-3]. The main advantages of a systematic point-of-care LUS approach during the surge are both a quick and reliable diagnosis of lung involvement at bedside, and a reduced exposure of other healthcare workers outside designated COVID-19 hospital areas. LUS has a great potential in the early identification of pneumonia [4]; however, LUS signs of COVID-19 pneumonia are non-specific and can be present in other respiratory conditions that commonly present to the Emergency Department (ED) [5, 6]. Recently, a simple approach based on the presence and distribution of a few basic LUS signs, determining patterns with different degrees of probability for COVID19 pneumonia, has been proposed [7-9]. This approach integrates clinical symptoms and the patient's medical history at presentation (clinical phenotypes) to provide context to the LUS exam.

The primary aim of our study was to describe the diagnostic accuracy of the combination of LUS probability patterns and clinical phenotypes in the prediction of the results of the reverse transcription-polymerase chain reaction (RT-PCR) test in a large international multicenter population suspected of COVID-19 during the pandemic surge. A second aim was to test an online training to implement a standardized LUS approach for COVID-19 in centers with different levels of LUS expertise. The study hypothesis is that a standardized LUS exam is feasible and reproducible, and can be useful for the early prediction of RT-PCR results in patients suspected of COVID-19.

\section{Methods}

\section{Study design and population}

This is a multicenter prospective observational study. From March 1st, 2020 until May 30th, 2020, patients from 20 different hospitals were evaluated (Online

\section{Take-home message}

In patients suspected for COVID-19, lung ultrasound patterns of probability integrated with patients' characteristics allow to rule in or rule out COVID-19 pneumonia at bedside with high accuracy, according to the different clinical phenotypes. This approach could support and expedite patients' management during a pandemic surge.

Resource 1). In each hospital, the main local LUS expert was nominated as the site principal investigator and was responsible for patient enrollment and LUS image acquisition.

Inclusion criteria were: (1) clinical suspicion of SARS$\mathrm{CoV}-2$ infection leading to presentation to the ED, or admission to COVID Unit or Intensive Care Unit (ICU) of the enrolling Hospitals. Clinical suspicion was based on the following symptoms lasting for at least 3 days in the absence of an alternative more probable diagnosis: major criteria (at least one): fever $>37.5^{\circ} \mathrm{C}$, cough, dyspnea, anosmia and/or ageusia; minor criteria (at least two): sore throat, bilateral conjunctivitis, generalized weakness, rhinorrhea, headache, diffuse musculoskeletal pain, gastrointestinal symptoms (diarrhea, nausea, vomiting); (2) age $\geq 18$ years. Exclusion criteria were: (1) a previous diagnosis of COVID-19 pneumonia; (2) a previous pneumonectomy, pleurodesis, or history of fibrothorax.

All the enrolled patients were examined by LUS and received a nasopharyngeal swab for SARS-CoV-2 RTPCR. Oxygen saturation and/or arterial blood gas analysis were measured in all patients.

\section{Ethical consideration}

The local Ethical Committee Boards approved the study, and the study was conducted following the ethical standards of local guidelines for good clinical practice. The list of ethical approvals is added as Online Resource 2. Informed consent was obtained in line with security protocols in place in the hospitals during the emergency, and depending on local rules and institutional regulations. 


\section{Clinical characteristics}

Each patient was characterized according to the presence of dyspnea, either objective or self-reported to the attending physician at admission. Desaturation and respiratory failure were defined as either a $\mathrm{PaO}_{2} / \mathrm{FiO}_{2}<300 \mathrm{mmHg}$ or Sat. $\mathrm{O}_{2} / \mathrm{FiO}_{2}<357$ [3]. Cardiopulmonary comorbidities were defined as a history of chronic respiratory and/ or cardiac disease, including severe chronic obstructive pulmonary disease, interstitial lung disease, primitive or metastatic lung cancer, heart failure, and cor pulmonale. These conditions are those that may create confounding LUS signs [4].

Three main different clinical phenotypes were identified and assigned to each patient at presentation, as previously described $[8,9]$. These phenotypes were defined as (1) mild phenotype in patients without dyspnea and/ or desaturation (mild non-respiratory symptoms and no signs of respiratory failure); (2) severe phenotype in patients with dyspnea and/or desaturation (dyspnea and/ or signs of respiratory failure); or (3) mixed phenotype in patients with cardiopulmonary comorbidities, irrespective of the clinical condition. From the severe group, we selected a subgroup with objective desaturation, as described above.

\section{Lung ultrasound}

A complete LUS examination was performed at presentation on the antero-lateral and posterior chest, as previously described $[8,9]$. Commercially available ultrasound equipments with convex transducers $(3.5-6.0 \mathrm{MHz})$ were used. The focus was placed on the pleural line. Depth was set at about $8-10 \mathrm{~cm}$, according to patient's size. Gain was regulated to optimize the image. The sonographers at each hospital were either ICU or ED clinicians, with documented previous experience in LUS in emergency and critical care. The number of operators was variable in each hospital; they were under the responsibility of the local principal investigator. Each LUS operator was blind to the result of RT-PCR test and any other imaging test or blood exam, but not to the patients' clinical condition. LUS was performed immediately at presentation whenever possible, always before the result of the RT-PCR test.

Each LUS examination was classified according to the following standardized, mutually exclusive, patterns $[8$, 9]:

- Low probability (LowLUS): a normal or near-normal LUS pattern characterized by bilateral A-lines with lung sliding and without significant B-lines.

- High probability (HighLUS): typical LUS pattern of COVID-19 pneumonia with bilateral and multifocal clusters of separated or coalescent B-lines, large hyperechoic bands (light beams), multifocal peripheral consolidations, regular and irregular pleural line, with or without large consolidations. These clusters should appear in patchy distribution, abruptly alternating with normal A-lines patterns ("spared areas").

- Intermediate probability (IntLUS): less typical pattern including unilateral isolated clusters of B-lines and light beams or focal multiple B-lines, with or without small peripheral consolidations.

- Alternative probability (AltLUS): a pattern more consistent with an alternative diagnosis such as an isolated large consolidation with dynamic air bronchograms (suggesting bacterial pneumonia) or without bronchograms (suggesting obstructive atelectasis), a large pleural effusion (either hydrostatic or inflammatory), diffuse homogeneously distributed B-lines (suggesting cardiogenic edema or diffuse fibrosis).

\section{Standardization of the LUS approach and inter-operator variability}

Before starting the enrollment, dedicated web meetings were organized to illustrate the protocol. Real cases were discussed to standardize LUS approach and pattern recognition. At the end of this preparatory phase, a video tutorial was sent to each site's principal investigator (Online Resource 3).

To assess LUS inter-operator variability in the interpretation of LUS patterns and signs, an online training exercise was set up, with a total of 53 clips including the whole range of significant LUS signs (separated B-lines, coalescent B-lines, light beams, large consolidations, small peripheral consolidations, pleural line alterations, and no alterations). Additionally, a group of eight clinical cases showing all 4 LUS patterns were presented. All the principal investigators were asked to recognize the main abnormalities in each video and establish the LUS pattern probability, blinded to any clinical information. If intraclass correlation coefficient (ICC) was $>0.8$, the sonographer was considered independent in LUS evaluation.

\section{Statistical analysis}

Continuous variables are expressed as mean \pm standard deviation. Two-sample comparisons were performed using the Mann-Whitney $U$ test, and the Chi-squared test for categorical data. Sensitivity, specificity, positive and negative predictive values, and accuracy were calculated using RT-PCR as the gold standard, and either the HighLUS as positive LUS exam and LowLUS/IntLUS/ AltLUS as negative exam (Model 1), or HighLUS and IntLUS as positive exam and LowLUS/AltLUS as negative exam (Model 2).

The association of selected variables with the positivity of RT-PCR was then assessed by logistic regression 
model using multivariable procedures (forward selection). Variables were selected for the univariate analysis according to the clinical probability of being associated with the positivity of RT-PCR; all variables with an associated $p$ value $<0.10$ at univariate analysis were included in the final multivariate analysis. Variance inflation factor (VIF) $>5$ was used to exclude variables due to multicollinearity. The interobserver variability in LUS assessment was examined by ICC and calculated in the whole series of paired measurements made by the participating Center principal investigators and the main expert sonographer. A $p$ value of $<0.05$ was considered statistically significant. All statistical analyses were performed using the Statistical Package for the Social Sciences (SPSS Inc., Chicago, Illinois, USA, version 20) and R software (R Foundation for Statistical Computing software).

\section{Results}

An initial number of 2035 patients were screened, and a final total number of 1462 patients were enrolled: 1022 patients had a positive RT-PCR and 440 a negative RTPCR (prevalence of COVID-19 at presentation of 69.8\%). The flowchart of patients is shown in Online Resource 4. Patients' characteristics are listed in Table 1. Seventyfour percent of the population was enrolled in EDs, 16\% in COVID units, and 10\% in ICUs.

The prevalence of the four different LUS patterns are shown according to RT-PCR positivity in the overall population and in the different clinical phenotypes (Online Resource 5). Table 2 reports LUS diagnostic accuracy in all subgroups, considering as positive LUS exam HighLUS alone or HighLUS and IntLUS. An example of the 4 LUS probability patterns is shown in Fig. 1. Data on the prevalence of the light beam (as a binary variable) in the different clinical subgroups are shown in Online Resource 6.

A multivariate logistic regression analysis was performed to assess the independent role of selected variables to predict a positive RT-PCR. Both the HighLUS and the light beam were predictive of a positive RT-PCR, independently of desaturation (Online Resource 7). No severe multicollinearity was found between dyspnea and respiratory failure $(\mathrm{VIF}=1.32)$ and between light beam and HighLUS $(V=2.95)$.

Respiratory failure was more prevalent in patients with the HighLUS compared to other LUS patterns $(65.5 \%$ vs $50.5 \%$ in AltLUS, $47.6 \%$ in IntLUS and $5.8 \%$ in LowLUS, Chi-squared $=252, p<0.0001$ ).
Table 1 Patients' characteristics according to RT-PCR positivity

\begin{tabular}{|c|c|c|c|}
\hline & $\begin{array}{l}\text { RT-PCR } \\
\text { negative } \\
(n=440)\end{array}$ & $\begin{array}{l}\text { RT-PCR } \\
\text { positive } \\
(n=1022)\end{array}$ & $p$ value \\
\hline Age (years) & $56.1 \pm 20.5$ & $62.2 \pm 16.4$ & $<0.0001$ \\
\hline Days from symptoms onset & $7.2 \pm 9.4$ & $9.0 \pm 7$ & $<0.0001$ \\
\hline $\begin{array}{l}\text { Cardiopulmonary comorbidi- } \\
\text { ties }(n)\end{array}$ & $128(29.1 \%)$ & 207 (20.3\%) & $<0.0001$ \\
\hline Dyspnea $(n)$ & 199 (45.2\%) & $700(68.5 \%)$ & $<0.0001$ \\
\hline Mild phenotype $(n=402)$ & $180(44.8 \%)$ & $222(55.2 \%)$ & $<0.0001$ \\
\hline Mixed phenotype $(n=335)$ & $128(38.2 \%)$ & $207(61.8 \%)$ & $<0.0001$ \\
\hline Severe phenotype $(n=725)$ & $132(18.2 \%)$ & $593(81.8 \%)$ & $<0.0001$ \\
\hline $\begin{array}{l}\text { Respiratory failure in the } \\
\text { severe phenotype }(n=497)\end{array}$ & $62(12.5 \%)$ & $435(87.5 \%)$ & $<0.0001$ \\
\hline
\end{tabular}

$R T-P C R$ reverse transcriptase-polymerase chain reaction

A total of 75 sonographers were involved in the study. The average overall agreement on the training video-clips was $90.6 \%$. The ICC of the whole series between each participating center principal investigator and the expert sonographer is listed in Online Resource 8. The average ICC was 0.980 (range 0.939-1.0); all sonographers showed an ICC $>0.90$. In a fixed-effects model including the center as a predictor, no relevant changes were observed in the coefficients and their significance, neither in the univariate analysis, nor in the multivariableadjusted model.

\section{Discussion}

Our findings show that some combinations between LUS patterns of probability for COVID-19 pneumonia and the clinical phenotype at presentation are accurate in predicting the result of the RT-PCR in patients with suspected SARS-CoV-2 infection. This standardized LUS protocol has shown low inter-operator variability among the US and European centers involved. Figure 2 reports the proposed operative algorithm based on LUS signs, and Fig. 3 details the main practical conclusions of our study.

To the best of our knowledge, this is the first large multicenter study describing the application of a standardized LUS protocol that combines the sonographic probability of pneumonia with the clinical phenotypes for a rapid decision-making and resource allocation in patients suspected of COVID-19. The role of LUS in patients with suspected COVID-19 is to identify an associated interstitial pneumonia, the 


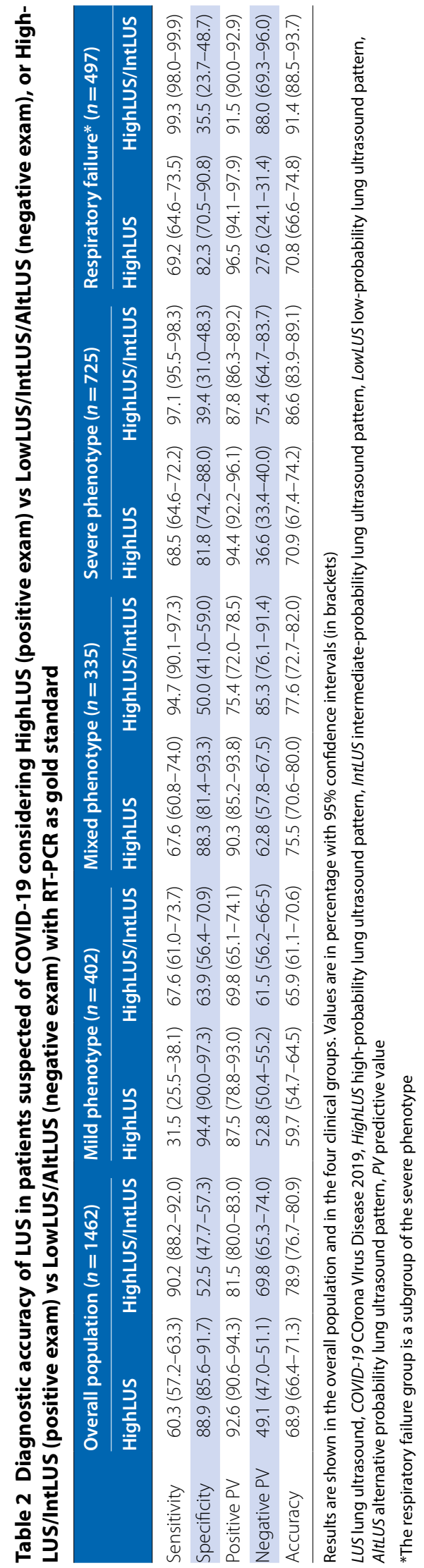

most common complication, and not to diagnose the infection, because pneumonia does not manifest in all patients with SARS-CoV-2 infection. Moreover, all the LUS signs of COVID-19 pneumonia are non-specific and may be present in other pulmonary conditions. These are the reasons why combining LUS patterns of probability for interstitial pneumonia with clinical phenotypes at presentation provides a structured guidance to the sonographer for a more effective and clinicallyoriented use of LUS.

HighLUS: in times of high prevalence of the disease, the HighLUS is highly predictive of COVID-19 pneumonia. In our population, the percentage of discordant cases showing HighLUS and negative RT-PCR was low and might be explained by the non-negligible rate of false-negative swab tests. Indeed, the first RT-PCR is limited by a rather low sensitivity and only repeated swabs, or bronchoalveolar lavage in some cases, may correctly allocate the diagnosis in those patients with a first falsenegative swab [10-12]. However, the possibility that an RT-PCR shifts to positivity after hospitalization and a reasonable incubation period always raises the suspicion of cross-infection; moreover, it is common experience that hospitalized patients with a typical clinical picture and suggestive computed tomography $(\mathrm{CT})$ pattern but repeatedly negative RT-PCR have been considered and treated as COVID-19 pneumonia, particularly in the peak of the COVID-19 surge [10]. Notwithstanding these limitations when the prevalence of the disease is high, detection of HighLUS in patients suspected for COVID19 predicts SARS-CoV-2 infection in all clinical subgroups, with a higher specificity in the mild phenotype.

The light beam, a relevant sign in the definition of the HighLUS, is also a strong independent predictor of RTPCR positivity. We speculate that this sign can be the ultrasound representation of the very early interstitial involvement corresponding to the ground-glass opacities that are typically visible on CT studies in the lung periphery during the initial phase of the disease (Fig. 4) [13-15]. The light beam is not specific for COVID-19, as it can be seen in other conditions not necessarily COVID-related; however, it is a typical feature of the LUS pattern in COVID-19 pneumonia, and its presence during a pandemic surge should prompt high suspicion for COVID19 pulmonary involvement.

IntLUS: as expected, the percentage of patients with IntLUS and negative RT-PCR was higher than in patients with HighLUS. However, the two patterns together achieved the highest sensitivity in all subgroups. Therefore, as a practical perspective, the absence of both HighLUS and IntLUS should be considered to rule out COVID-19 pneumonia with high sensitivity, which increases as the clinical situation worsens 
(from $90.2 \%$ in the overall population to $99.3 \%$ in respiratory failure).

LowLUS: overall, $35 \%$ of patients with the LowLUS had positive RT-PCR. This apparent discrepancy is explained by the presence of the virus in patients without pneumonia or with a very mild form of pulmonary involvement, invisible to LUS $[16,17]$. However, in the absence of sonographically detectable pulmonary involvement (A-lines pattern throughout), it is unlikely that the respiratory failure is related to COVID-19. Interestingly enough, some patients with severe phenotype and LowLUS showed a positive RT-PCR. A possible explanation for this discrepancy may be the subjective nature of the reported dyspnea, which could be linked to factors other than pneumonia, including the stressful situation for patients with suspected COVID-19. Another possibility is the profound muscle weakness and general sense of fatigue, frequently accompanying symptoms of this viral disease. Indeed, when the analysis was restricted to the subgroup of patients with objective desaturation (respiratory failure subgroup), no patient with LowLUS resulted as RT-PCR positive. Thus, our study demonstrated that LowLUS in patients with objective signs of respiratory failure allows to rule out COVID-19 as the main cause of respiratory symptoms, suggesting searching for an alternative diagnosis.

AltLUS: bacterial pneumonia, cardiogenic pulmonary edema, pulmonary fibrosis, and neoplastic or infective pleural effusion were the main causes for AltLUS [18]. In both the severe and mixed phenotype, the vast majority of patients showing the AltLUS was confirmed RT-PCR negative $(84.8 \%$ and $84.4 \%$, respectively). Thus, even in a moment of high prevalence of COVID-19, the AltLUS correctly predicted the negativity of RT-PCR in most patients. These findings are important for clinicians trying to differentiate between the exacerbation of a chronic disease and COVID-19 pneumonia.

Mixed phenotype: in the group of patients assigned to the "mixed" phenotype, the role of LUS is particularly challenging. Pattern recognition may become difficult for the possibility of exacerbations of pre-existing pulmonary alterations. Indeed, many pulmonary conditions, both acute and chronic, share similar LUS signs with
COVID-19 pneumonia. Despite these difficulties, the detection of the HighLUS in the mixed phenotype predicted a positive RT-PCR with good specificity. However, detection of IntLUS in patients with chronic pre-existing pulmonary diseases indicates the need for further testing.

LUS patterns and CT findings: our findings show the practical advantage of classifying LUS in four probability patterns within clinical phenotypes, which has not been used in previous LUS studies [17, 19-23]. The language used in this study to define the LUS patterns is similar to the language suggested for $\mathrm{CT}$ findings related to COVID-19 [8, 9, 13]. The "typical appearance" on CT can be assimilated to the HighLUS, the "indeterminate appearance" to IntLUS, the "atypical appearance" to AltLUS, and the "negative" to LowLUS. CT scan is the gold standard of chest imaging with a sensitivity superior to LUS. It is reasonable to describe LUS in terms of "probability", whereas CT findings are described as "appearance", and while the absence of pulmonary signs on CT indicates a "negative" study, the absence of LUS signs indicates "low probability" of COVID-19 pneumonia. We encourage a standard application of this language to LUS findings in the first approach to suspected cases. On the other hand, it is also evident the need to differentiate the main clinical phenotypes at presentation, considering that different combinations between clinical phenotypes and LUS patterns give different accuracy in predicting RT-PCR results. Indeed, the main principle of point-ofcare LUS still remains the need for a close correlation with the clinical condition of the patient [22].

\section{Limitations}

Our study is limited by the lack of a definitive gold standard. We did not perform a systematic comparison with CT scan to confirm LUS findings of pneumonia and did not perform a follow-up of patients for definitive confirmation of COVID-19 infection in doubtful cases. COVID-19 poses several diagnostic dilemmas: studies on LUS for COVID-19 should combine the need to confirm the infection, which cannot be pursued by chest imaging, and the need to detect pneumonia, which is in the power of LUS. The RT-PCR test is limited by a low sensitivity and delay in the results, but it is the standard to indicate

\footnotetext{
(See figure on next page.)

Fig. 1 Representative images of the four probability patterns in symptomatic patients, showing the distribution of the LUS signs of COVID-19 pneumonia. First row: bilateral distribution of typical LUS interstitial signs (high probability) in a case RT-PCR positive. Second row: monolateral distribution of interstitial LUS signs (intermediate probability) in a case RT-PCR positive. Third row: presence of atypical signs (alternative probability) in a case showing an isolated large consolidation with air bronchograms, due to bacterial pneumonia, and RT-PCR negative. Fourth row: absence of interstitial LUS signs (low probability) in a case RT-PCR negative. LUS lung ultrasound, COVID-19 Corona Virus Disease 2019, RT-PCR reverse transcriptase-polymerase chain reaction
} 


\section{High probability}

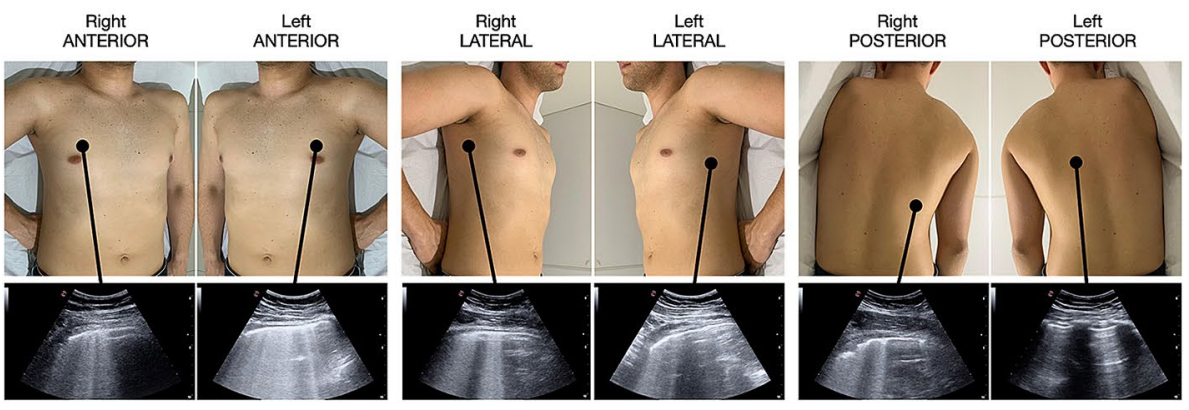

Intermediate probability
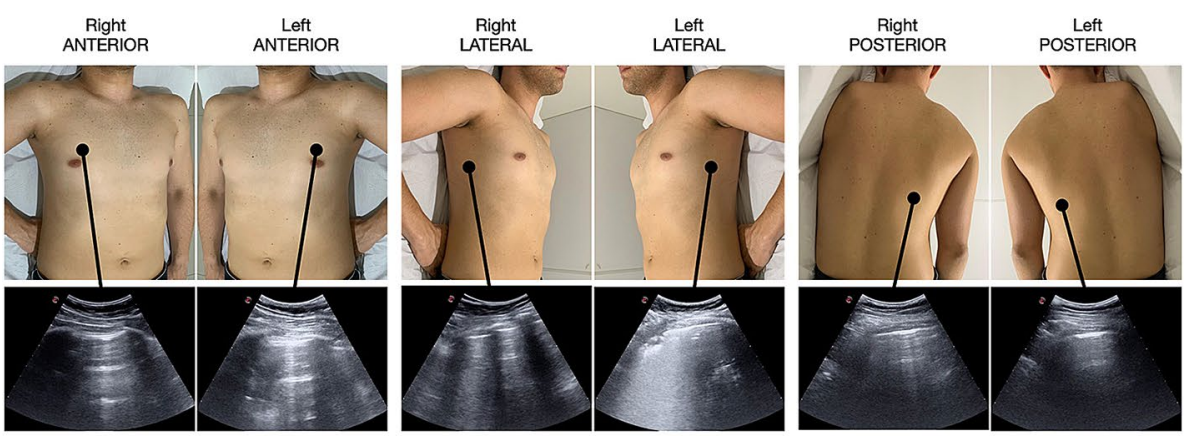

\section{Alternative pattern}
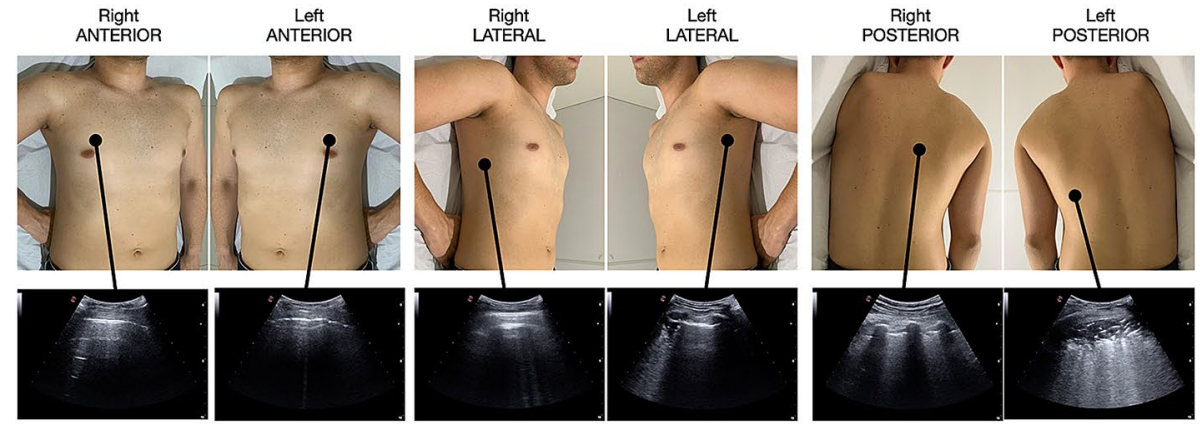

\section{Low probability}
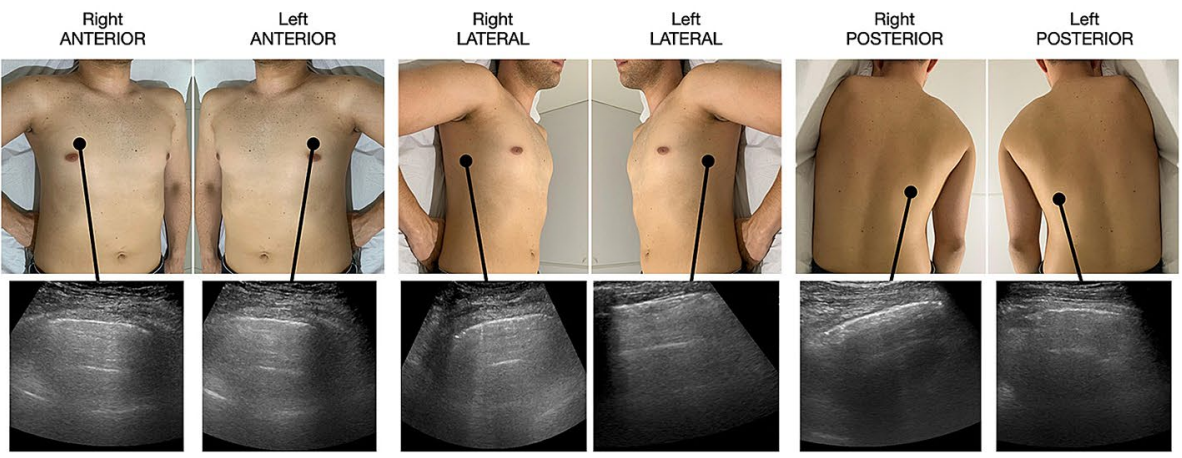

Fig. 1 (See legend on previous page.) 


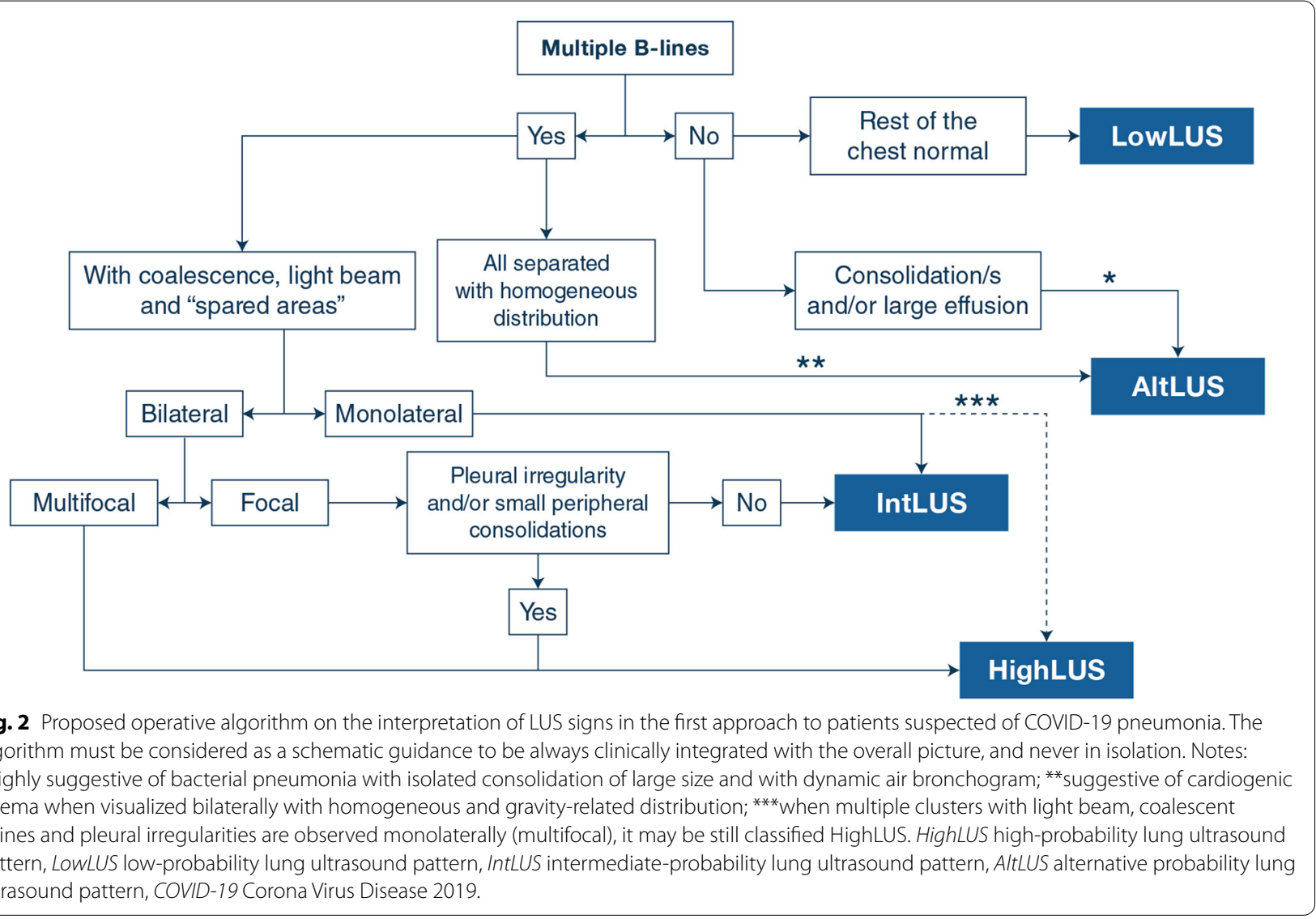

patients' first diagnosis and guide hospital allocation in the clinical practice; therefore, it is the reference we used to assess LUS accuracy.

The LUS operators were blind to the RT-PCR results but aware of the patients' clinical condition, which may have biased LUS interpretation. However, COVID-19 may occur with a wide variety of clinical pictures and the LUS diagnosis is not linked to the clinical severity and symptoms, minimizing this potential bias. Patients were enrolled when a sonographer with documented LUS experience was present; although this can also have introduced some selection bias, the selection was anyway completely random.

We assessed the inter-operator variability in the recognition of signs and patterns on clips. This is not the same as assessing LUS variability in the same patient, which was not feasible during the pandemic. However, our data show how a standardized LUS protocol may be implemented with high inter-center reproducibility and applied worldwide by operators with variable skills and experience.

While the number of severe pneumonias with respiratory failure is well estimated during the pandemic, the percentage of asymptomatic infections is undoubtedly underestimated. Even more, the number of interstitial pneumonias in mildly symptomatic or even asymptomatic patients is far from being fully investigated [24]. Future studies should address the real prevalence of pneumonia associated with COVID-19, and LUS can be the ideal sensitive tool to accomplish this task.

Finally, the interpretation of our results is linked to the prevalence of the disease, which was particularly high during the first pandemic wave. With a lower prevalence, the significance of these findings needs to be revisited.

\section{Conclusions}

A standardized LUS protocol combining ultrasound probability patterns with clinical phenotypes in patients suspected of COVID-19 pneumonia at presentation is feasible and reproducible. The HighLUS and IntLUS together are very sensitive in ruling out interstitial pneumonia, with increasing values with worsening clinical phenotypes; whereas in patients with mild symptoms, the HighLUS is very specific in ruling in interstitial pneumonia. The detection of the AltLUS suggests an alternative diagnosis. Our approach may be useful to rapidly guide management for wiser use of hospital resources during a 

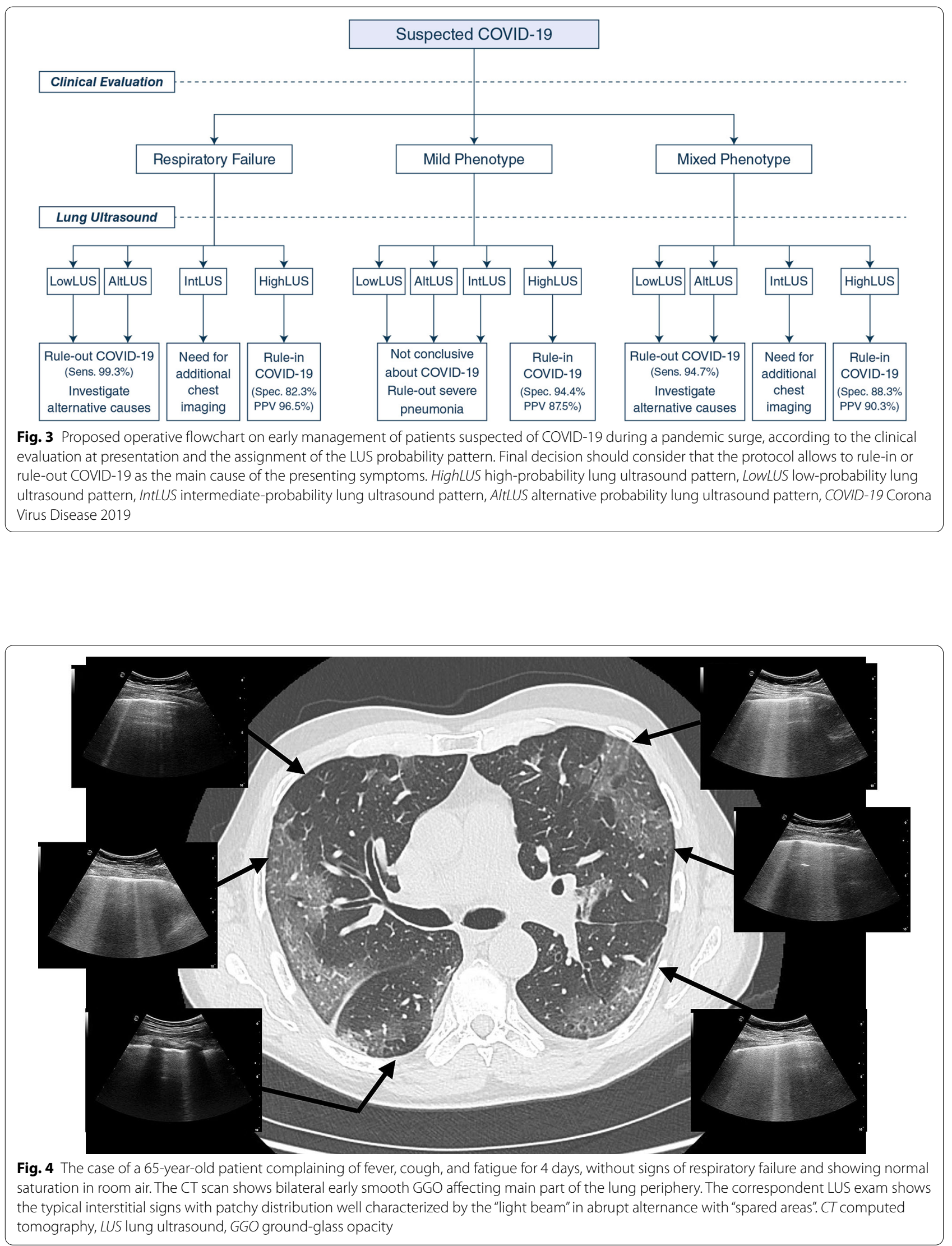
pandemic surge, and may even represent the only available diagnostic tool in scarce resource areas.

\section{Supplementary Information}

The online version contains supplementary material available at https://doi. org/10.1007/s00134-021-06373-7.

\section{Author details}

1 Department of Emergency Medicine, San Luigi Gonzaga University Hospital, Regione Gonzole 10, Orbassano, 10024 Turin, Italy. ${ }^{2}$ Institute of Clinical Physiology, National Research Council, Via Moruzzi 1, 56124 Pisa, Italy. ${ }^{3}$ Emergency Department, Department of Internal Medicine, IRCCS Policlinico San Matteo Foundation, University of Pavia, Pavia, Italy. ${ }^{4}$ Emergency Medicine Department, University of Pisa, Pisa, Italy. ${ }^{5}$ Emergency Department, IRCCS Azienda Ospedaliero-Universitaria di Bologna, Bologna, Italy. ${ }^{6}$ Internal Medicine Service, Infanta Cristina University Hospital, Madrid, Spain. ${ }^{7}$ University Hospital, Lleida, Spain. ${ }^{8}$ Emergency Department Parini Hospital, Aosta, Italy. ${ }^{9}$ Vita-Salute University, San Raffaele, Milan, Italy. ${ }^{10}$ Cardiovascular Imaging Unit, San Raffaele Hospital, Milan, Italy. ${ }^{11}$ Francisco de Vitoria University Hospital, Madrid, Spain. ${ }^{12}$ Chelsea and Westminster Hospital, London, UK. ${ }^{13}$ Department of Emergency Medicine, Careggi University Hospital, Florence, Italy. ${ }^{14}$ Department of Surgical, Medical and Molecular Pathology and Critical Care Medicine, University of Pisa, Pisa, Italy. ${ }^{15}$ Department of Anesthesiology, Ente Ospedaliero Ospedali Galliera, Genoa, Italy. ${ }^{16}$ Ferrero Hospital, Verduno, Italy. ${ }^{17}$ Carolinas Medical Center, Atrium Health, Charlotte, USA. ${ }^{18}$ Intensive Care Unit Department, University of Padova, Padova, Italy. ${ }^{19}$ Department of Emergency Medicine, Indiana University, Indianapolis, Indiana, USA. ${ }^{20}$ San Giovanni Bosco Hospital, Turin, Italy. ${ }^{21}$ Saint Bartholomew's Hospital, London, UK. ${ }^{22}$ Frimley Health Foundation Trust, Frimley, UK. ${ }^{23}$ Emergency Department, Spedali Civili Hospital, Brescia, Italy. ${ }^{24}$ Department of Medicine, University Hospital of Udine, Udine, Italy. ${ }^{25}$ Mater University Hospital, Dublin, Republic of Ireland. ${ }^{26}$ Anesthesiology Service, University Hospital of Puerta del Mar, Cadiz, Spain. ${ }^{27}$ Department of Political Sciences, University of Pisa, Pisa, Italy.

\section{Acknowledgements}

We are deeply thankful to all the Doctors, Nurses and Healthcare Personnel, who have been risking their lives to assist and cure these patients. We would like to thank also Laura Carrozzi, Giulia Palmisano, Valentina Poli, Francesca Vitale, and Manuela Toscano for their support with the management of patients and LUS examinations. The members of the International Multicenter Study Group on LUS in COVID-19 Collaborators are: Thomas Fraccalini: Department of Emergency Medicine, San Luigi Gonzaga University Hospital, Torino, Italy; Alessandro Vendrame: Department of Emergency Medicine, San Luigi Gonzaga University Hospital, Torino, Italy; Vittoria Basile: Department of Emergency Medicine, San Luigi Gonzaga University Hospital, Torino, Italy; Alessandro Cipriano: Emergency Medicine Department, University of Pisa, Italy; Francesca Frassi: Emergency Medicine Department, University of Pisa, Italy; Massimo Santini: Emergency Medicine Department, University of Pisa, Italy; Marco Falcone: Infectious Diseases Unit, University of Pisa, Italy; Francesco Menichetti: Infectious Diseases Unit, University of Pisa, Italy; Bruno Barcella: Emergency Department, Department of Internal Medicine, IRCCS Policlinico San Matteo Foundation, University of Pavia, Italy; Marzia Delorenzo: Emergency Department, Department of Internal Medicine, IRCCS Policlinico San Matteo Foundation, University of Pavia, Italy; Flavia Resta: Emergency Department, Department of Internal Medicine, IRCCS Policlinico San Matteo Foundation, University of Pavia, Italy; Giulia Vezzoni: Emergency Department, Department of Internal Medicine, IRCCS Policlinico San Matteo Foundation, University of Pavia, Italy; Marco Bonzano: Emergency Department, Department of Internal Medicine, IRCCS Policlinico San Matteo Foundation, University of Pavia, Italy; Domenica Federica Briganti: Emergency Department, Department of Internal Medicine, IRCCS Policlinico San Matteo Foundation, University of Pavia, Italy; Giovanni Cappa: Emergency Department, Department of Internal Medicine, IRCCS Policlinico San Matteo Foundation, University of Pavia, Italy; Ilaria Zunino: Emergency Department, Department of Internal Medicine, IRCCS Policlinico San Matteo Foundation, University of Pavia, Italy; Lorenzo Demitry: Emergency Department, Department of Internal Medicine, IRCCS Policlinico San Matteo Foundation, University of Pavia, Italy; Damiano Vignaroli: Emergency Department, Department of Internal Medicine, IRCCS Policlinico San Matteo Foundation, University of Pavia, Italy; Lorenzo Scattaglia and Santi Di Pietro: Emergency Department, Department of Internal Medicine, IRCCS Policlinico San Matteo Foundation, University of Pavia, Italy; Marco Bazzini: Emergency Department, Department of Internal Medicine, IRCCS Policlinico San Matteo Foundation, University of Pavia, Italy; Vincenzo Capozza: Emergency Department, Department of Internal Medicine, IRCCS Policlinico San Matteo Foundation, University of Pavia, Italy; María Mateos González: Internal Medicine Service, Infanta Cristina University Hospital, Madrid, Spain; Rosa Vilella Gibal: University Hospital, Lleida, Spain; Ramon Piñol Ibarz: University Hospital, Lleida, Spain; Luis Martin Alfaro: University Hospital, Lleida, Spain; Carlos Martin Alfaro: University Hospital, Lleida, Spain; Maria Galindo Alins: University Hospital, Lleida, Spain; Alice Brown: Chelsea and Westminster Hospital, London, UK; Hannah Dunlop: Chelsea and Westminster Hospital, London, UK; Maria Luisa Ralli: Department of Emergency Medicine, Careggi University Hospital, Firenze, Italy; Paolo Persona: Intensive Care Unit Department, University of Padova, Italy; Frances M. Russel: Department of Emergency Medicine, Indiana University, USA; Peter S. Pang: Department of Emergency Medicine, Indiana University, USA; Serena Rovida: Royal London Hospital, London, UK; Cristian Deana: Department of Medicine, University Hospital of Udine, Italy; Diego Franchini: Emergency Department, Spedali Civili Hospital, Brescia, Italy. Funding

No funding sources were used in the study.

\section{Compliance with ethical standards}

\section{Conflicts of interest}

All the authors have had access to all the data of the study and accept responsibility for its validity. None of the authors and collaborators has conflicts of interest related to the present paper to diclose.

\section{Publisher's Note}

Springer Nature remains neutral with regard to jurisdictional claims in published maps and institutional affiliations.

Received: 16 December 2020 Accepted: 19 February 2021

Published online: 20 March 2021

\section{References}

1. Lichter Y, Topilsky Y, Taieb P, Banai A, Hochstadt A, Merdler I, Gal Oz A, Vine J, Goren O, Cohen B, Sapir O, Granot Y, Mann T, Friedman S, Angel Y, Adi N, Laufer-Perl M, Ingbir M, Arbel Y, Matot I, Szekely Y (2020) Lung ultrasound predicts clinical course and outcomes in COVID-19 patients. Intensive Care Med 46:1873-1883

2. de Almeida Monteiro RA, Duarte-Neto AN, Ferraz da Silva LF, de Oliveira EP, do Nascimento ECT, Mauad T, Saldiva PHDN, Dolhnikoff M (2021) Ultrasound assessment of pulmonary fibroproliferative changes in severe COVID-19: a quantitative correlation study with histopathological findings. Intensive Care Med 3:1-9. https://doi.org/10.1007/ s00134-020-06328-4

3. Zieleskiewicz L, Markarian T, Lopez A, Taguet C, Mohammedi N, Boucekine M, Baumstarck K, Besch G, Mathon G, Duclos G, Bouvet L, Michelet P, Allaouchiche B, Chaumoître K, Di Bisceglie M, Leone M, Network AZUREA (2020) Comparative study of lung ultrasound and chest computed tomography scan in the assessment of severity of confirmed COVID-19 pneumonia. Intensive Care Med 46:1707-1713

4. Volpicelli G, Elbarbary M, Blaivas M, Lichtenstein DA, Mathis G, Kirkpatrick AW, Melniker L, Gargani L, Noble VE, Via G, Dean A, Tsung JW, Soldati G, Copetti R, Bouhemad B, Reissig A, Agricola E, Rouby JJ, Arbelot C, Liteplo A, Sargsyan A, Silva F, Hoppmann R, Breitkreutz R, Seibel A, Neri L, Storti E, Petrovic T, International Liaison Committee on Lung Ultrasound (ILC-LUS) for International Consensus Conference on Lung Ultrasound (ICC-LUS) (2012) International evidence-based recommendations for point-of-care lung ultrasound. Intensive Care Med 38:577-591

5. Laursen CB, Sloth E, Lassen AT, Christensen Rd, Lambrechtsen J, Madsen PH, Henriksen DP, Davidsen JR, Rasmussen F (2014) Point-of-care ultrasonography in patients admitted with respiratory symptoms: a singleblind, randomised controlled trial. Lancet Respir Med 2:638-646 
6. Gargani L, Soliman-Aboumarie H, Volpicelli G, Corradi F, Pastore MC, Cameli M (2020) Why, when, and how to use lung ultrasound during the COVID-19 pandemic: enthusiasm and caution. Eur Heart J CardiovasC Imaging 21:941-948

7. Volpicelli G, Gargani L (2020) Sonographic signs and patterns of COVID-19 pneumonia. Ultrasound J 12:22. https://doi.org/10.1186/ s13089-020-00171-W

8. Volpicelli G, Lamorte A, Villén T (2020) What's new in lung ultrasound during the COVID-19 pandemic. Intensive Care Med 46:1445-1448

9. Millington SJ, Koenig S, Mayo P, Volpicelli G (2021) Lung ultrasound for patients with coronavirus disease 2019 pulmonary disease. Chest 159:205-211

10. Ai T, Yang Z, Hou H, Zhan C, Chen C, Lv W, Tao Q, Sun Z, Xia L (2020) Correlation of chest CT and RT-PCR testing for coronavirus disease 2019 (COVID-19) in China: a report of 1014 cases. Radiology 296:E32-E40

11. Li Y, Yao L, Li J, Chen L, Song Y, Cai Z, Yang C (2020) Stability issues of RTPCR testing of SARS-CoV-2 for hospitalized patients clinically diagnosed with COVID-19. J Med Virol 92:903-908

12. Arevalo-Rodriguez I, Buitrago-Garcia D, Simancas-Racines D, ZambranoAchig P, Del Campo R, Ciapponi A, Sued O, Martínez-García L, Rutjes A Low N, Bossuyt PM, Perez-Molina JA, Zamora J (2020) False-negative results of initial RT-PCR assays for COVID-19: a systematic review. https:// www.medrxiv.org/content/10.1101/2020.04.16.20066787v1

13. Simpson S, Kay FU, Abbara S, Bhalla S, Chung JH, Chung M, Henry TS, Kanne JP, Kligerman S, Ko JP, Litt H (2020) Radiological Society of North America Expert Consensus Statement on reporting chest CT findings related to COVID-19. Endorsed by the Society of Thoracic Radiology, the American College of Radiology, and RSNA. J Thorac Imaging 35:219-227

14. Revel MP, Parkar AP, Prosch H, Silva M, Sverzellati N, Gleeson F, Brady A, European Society of Radiology (ESR), the European Society of Thoracic Imaging (ESTI) (2020) COVID-19 patients and the radiology department - advice from the European Society of Radiology (ESR) and the European Society of Thoracic Imaging (ESTI). Eur Radiol 30:4903-4909

15. Han R, Huang L, Jiang H, Dong J, Peng H, Zhang D (2020) Early clinical and CT manifestations of coronavirus disease 2019 (COVID-19) pneumonia. AJR Am J Roentgenol 215:338-343

16. Nazerian P, Volpicelli G, Vanni S, Gigli C, Betti L, Bartolucci M, Zanobetti M, Ermini FR, lannello C, Grifoni S (2015) Accuracy of lung ultrasound for the diagnosis of consolidations when compared to chest computed tomography. Am J Emerg Med 33:620-625

17. Colombi D, Petrini M, Maffi G, Villani GD, Bodini FC, Morelli N, Milanese G, Silva M, Sverzellati N, Michieletti E (2020) Comparison of admission chest computed tomography and lung ultrasound performance for diagnosis of COVID-19 pneumonia in populations with different disease prevalence. Eur J Radiol 133:109344. https://doi.org/10.1016/j.ejrad.2020. 109344

18. Gargani L, Volpicelli G (2014) How I do it: lung ultrasound. Cardiovasc Ultrasound 2:25. https://doi.org/10.1186/1476-7120-12-25

19. Soldati G, Smargiassi A, Inchingolo R, Buonsenso D, Perrone T, Briganti DF, Perlini S, Torri E, Mariani A, Mossolani EE, Tursi F, Mento F, Demi L (2020) Proposal for international standardization of the use of lung ultrasound for patients with COVID-19: a simple, quantitative, reproducible method. J Ultrasound Med 39:1413-1419

20. Haak SL, Renken IJ, Jager LC, Lameijer H, van der Kolk BBY (2020) Diagnostic accuracy of point-of-care lung ultrasound in COVID-19. Emerg Med J. https://doi.org/10.1136/emermed-2020-210125

21. Sorlini C, Femia M, Nattino G, Bellone P, Gesu E, Francione P, Paternò M, Grillo P, Ruffino A, Bertolini G, Cariati M, Cortellaro F, Fenice Network (Italian Group for Clinical Research in Emergency Medicine) (2020) The role of lung ultrasound as a frontline diagnostic tool in the era of COVID-19 outbreak. Intern Emerg Med. https://doi.org/10.1007/s11739-020-02524-8

22. Pivetta E, Goffi A, Tizzani M, Locatelli SM, Porrino G, Losano I, Leone D, Calzolari G, Vesan M, Steri F, Ardito A, Capuano M, Maria G, Giulia S, Dutto S, Avolio M, Cavallo R, Bartalucci A, Paglieri C, Morello F, Richiardi L, Maule M, Lupia E (2020) Lung ultrasound for the diagnosis of SARS-CoV-2 pneumonia in the Emergency Department. Ann Emerg Med. https://doi.org/ 10.1016/j.annemergmed.2020.10.008

23. Lieveld AWE, Kok B, Schuit FH, Azijli K, Heijmans J, van Laarhoven A, Assman NL, Kootte RS, Olgers TJ, Nanayakkara PWB, Bosch FH (2020) Diagnosing COVID-19 pneumonia in a pandemic setting: Lung Ultrasound versus CT (LUVCT) a multi-centre, prospective, observational study. ERJ Open Res. https://doi.org/10.1183/23120541.00539-2020

24. Shi H, Han X, Jiang N, Cao Y, Alwalid O, Gu J, Fan Y, Zheng C (2020) Radiological findings from 81 patients with COVID-19 pneumonia in Wuhan, China: a descriptive study. Lancet Infect Dis 20:425-434 